\title{
A Fast, Highly Sensitive and Cheap Tool for Mutation Analysis of Complex Genes
}

\author{
Norbert Arnold ${ }^{\#}$, Eva Groß, Ulrike Schwarz- \\ Boeger, Jacobus Pfisterer, Walter Jonat and \\ Marion Kiechle \\ Department of Gynecology and Obstetrics, \\ Christian-Albrechts-Universität, Kiel, \\ Germany
}

The mutation analysis of complex genes without hot spots for sequence variation is very time consuming and expensive. The aim of our study was to introduce the Denaturing highperformance liquid chromatography (DHPLC) technique into the repertoire of scanning methods for DNA mutations and polymorphisms in the BRCA1 gene. To assess the accuracy of this screening method the analysis was done in parallel with direct sequencing. With 33 fragments we were able to screen the whole coding sequence and exon/intron boundaries of the BRCA1 gene.

The comparison of the results revealed that with DHPLC none of the alterations detected by direct sequencing was missed. Neither false positive signals nor false negative results were obtained. In cases of described genetic variations the nature of the alteration could be predicted independently of sequencing information, due to their difference in retention times. This could be demonstrated best in overlaying the obtained profiles of the test samples with the profiles of the reference fragments. In a blinded study one group predicted the nature of occuring sequence variations which was than compared with the sequencing results done by another group. Up to now no discrepancy has occured.

The mutation detection of BRCA1 and other genes can be simplified tremendously and speeded-up by using the DHPLC technique. So far it is the only prescreening method which allows a prediction of sequence alterations and may discriminate between mutations and polymorphisms. In comparison to direct sequencing, the costs of DHPLC are 30 times lower and BRCA1 mutation results can be obtained within two days.

\footnotetext{
\# Correspondence: Arnold Norbert, Department of Gynecology and Obstetrics, Michaelisstr. 16, D-24105 Kiel, Germany, Tel.: +49 431597 2173, Fax: +49 431 597 2539, E-mail: nkarnold@email.uni-kiel.de.
} 


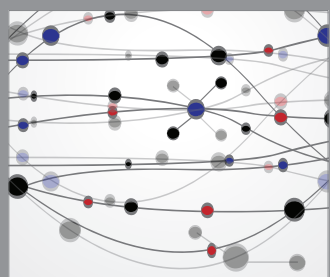

The Scientific World Journal
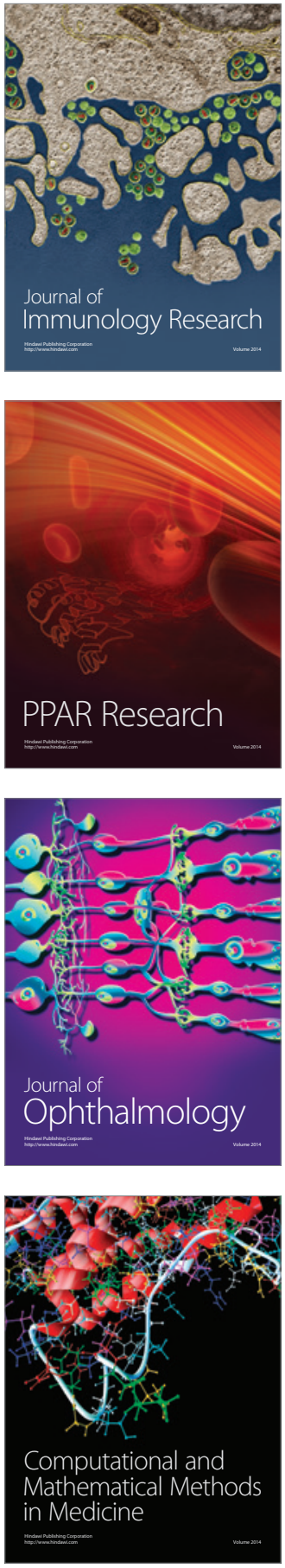

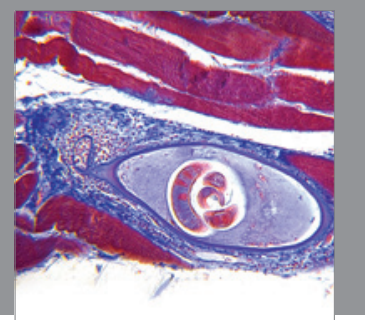

Gastroenterology

Research and Practice
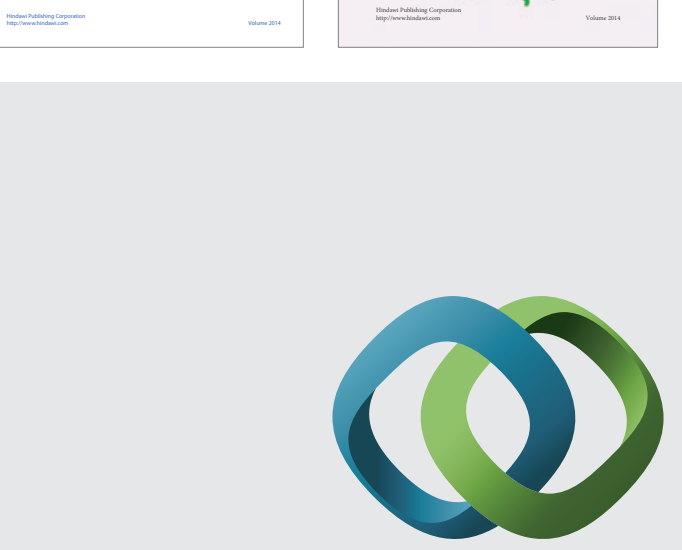

\section{Hindawi}

Submit your manuscripts at

http://www.hindawi.com
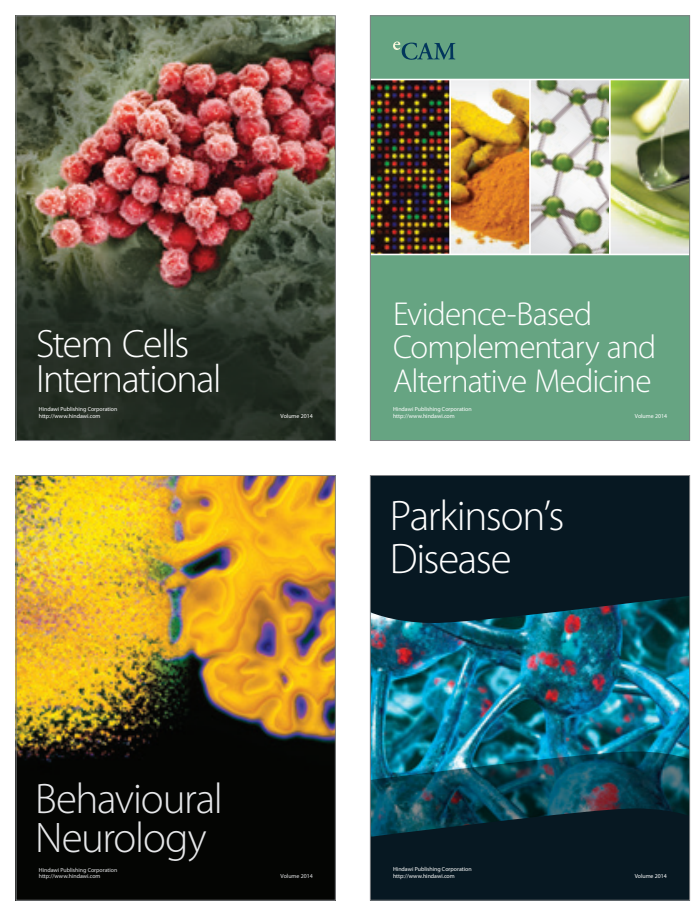

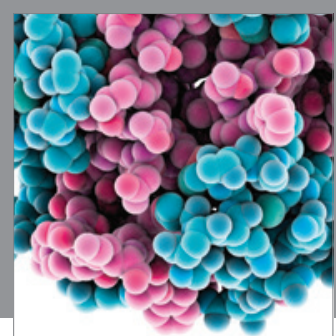

Journal of
Diabetes Research

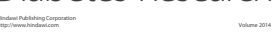

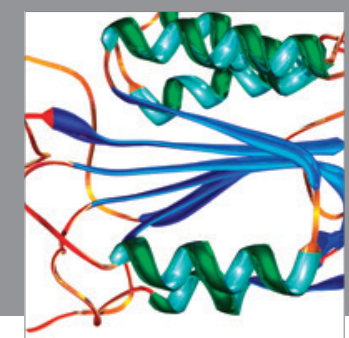

Disease Markers
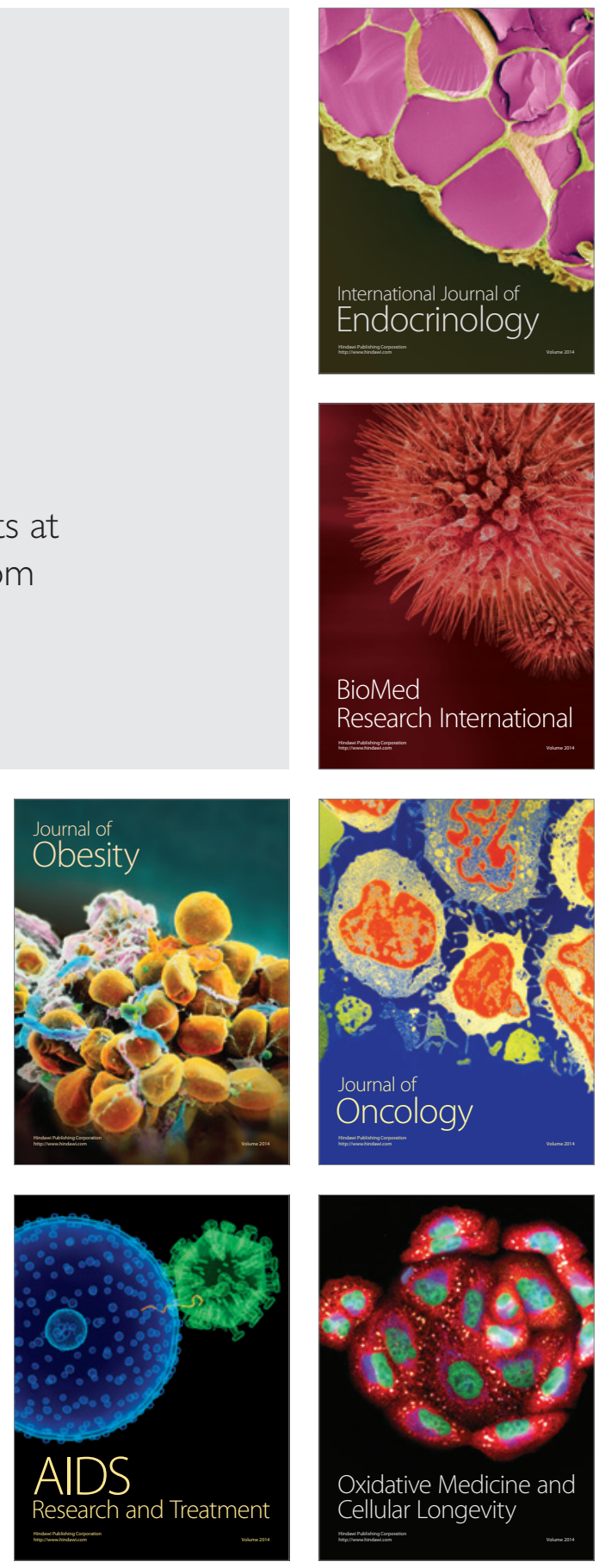